\title{
Research proposal (DRX)
}

\author{
Mahmud Matin ${ }^{1}$ \\ ${ }^{1}$ Affiliation not available
}

May 5, 2020

Keywords: DRX(Discontinuous Reception), LTE(Long Term Evaluation), CQI(Channel Quality Indicator), UE(User Equipment).

\section{Literature review}

Long Term Evolution(LTE)of Universal Mobile Telecommunications System (UMTS)offers high bandwidth for data transfer and lower latencies so that the system can support various types of application but wireless device batteries are still the same size.LTE has a downlink data rate of 100Mbps and uplink data rate of $50 \mathrm{Mbps}$.Some real-time and delay sensitive applications, such as video streaming, mobile TV and multimedia online gaming that is further enabled by LTE. This types of high data rate operation can be achieved by applying various methods [4].So the main purpose of this situation is to overcome the battery consumption problem and device lifetime length additionally it needs to be optimized for network load. For this purpose, the 3GPP Long-Term Evolution(LTE)standard has specified new discontinuous reception(DRX)based on the Universal Mobile Telecommunications System(UMTS)DRX mechanism, called, LTE-DRX, idle/sleep modes in WiMAX, introduced to improve UE battery lifetime [1][2][3][4].

The 3rd Generation Partner research(3GPP) initially incorporated DRX during their Release 8 documentation. At that time, the widely implemented mobile network was the UMTS network or the $3 \mathrm{G}$ mobile network in which DRX was being put into operation to prolong the battery lifetime. DRX and RRC (Radio Resource Control) mechanisms will operate in different forms, which depends on the characteristics of the traffic. Consequently, it has an impact on the UE's power consumption. For an effective investigation on the sufficiency of the LTE DRX mechanisms, traffic analysis is of high importance. Thus they studied the characteristics of different types of services. They introduced the DRX operation under different RRC states. According to, RRC states has dramatically impacted on UE's power consumption and packet transmission delay. For the performance of DRX, several analytical studies have been conducted [5].

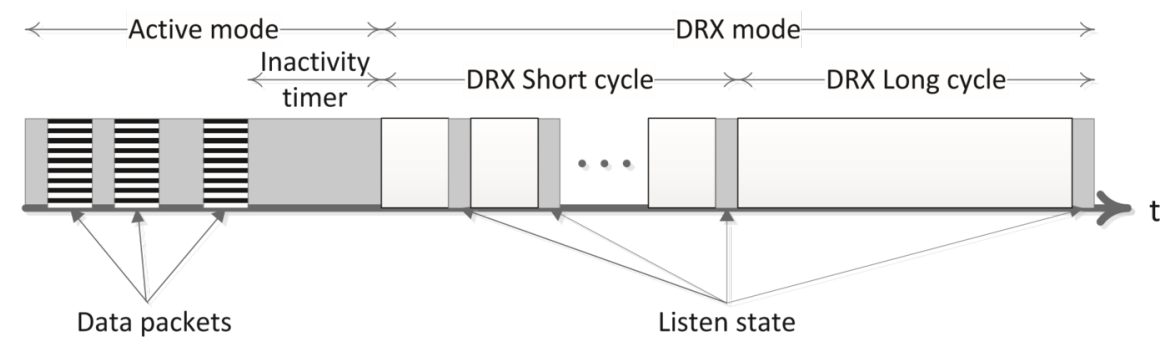

Active state

Sleep state

Figure 1: DRX in wireless network [6]. 
In the LTE DRX mechanism, the sleep/wake scheduling of each UE is determined by the following four parameters DRX Short Cycle, DRX Long Cycle, DRX Inactivity Timer, and DRX Short Cycle Timer. In LTE DRX, the sleep/wake-up mode consists of the three different states, namely, Inactivity period, Light Sleep period, and Deep Sleep period. The transition from the Inactivity period to the Light Sleep period is controlled [7].

\section{Active state/On state}

When a UE is actively monitoring the RF spectrum for data frames.

- Active Time: The time data packets are received by a UE.

- DRX Inactivity Timer: A timer used to initiate the sleep states.

\section{Sleep state/Off state}

When a UE has powered down and not monitoring the RF spectrum.

- Listen state: A very short period of time when the UE powers up during DRX short cycle to monitor the RF spectrum for incoming Physical Downlink Control Channel (PDCCH)transmissions.DRX Short Cycle: A period of time when the UE is in sleep state but periodically transitions to the listen state to monitor the PDCCH for incoming data frames.

- DRX Short Cycle Timer: During the DRX short cycle the DRX Short Cycle Timer sets off the listen state.

- DRX Long Cycle: A time span longer than the DRX Short Cycle where an UE stays in the sleep state and wakes up only at the end of the cycle to monitor the PDCCH.

- DRX Long Cycle Timer: During the DRX long cycle the DRX long Cycle Timer set off the listen state [1].

- DL-related scheduling messages These messages are needed for a scheduled UE to be able to properly receive, decode and demodulate DLSCH. Moreover, information about DL-SCH resource allocation, transport format, and information about hybrid ARQ are also included [8].

- UL-related scheduling messages These messages contain scheduling grant information. These are intended to inform a scheduled UE what uplink resources and transport format to use for UL-SCH. The PDCCH is located in the first (up to three) OFDM symbols in the first slot of a subframe [5].

- SR-pending timer According to the specifications, a scheduling request shall be considered pending until UL grant is received and UE shall monitor the PDCCH whenever the UL grant is expected. This functionality is implemented using SR-pending Timer in the System Simulator. SR-pending Timer is triggered ON whenever a scheduling request is made by UE and it keeps the UE awake as long as it has received the UL grant from the eNB.The duration of this timer is flexible in the sense that it could be as short as one TTI during low traffic load scenario where UE actually can receive a grant in $1 \mathrm{~ms}$. Whereas, in cases of high traffic load, it may take as many as several TTIs for UE to receive the uplink grant which results in longer awake time. Since SR-pending Timer is independent of On-duration Timer and any UE can send a scheduling request independent of its DRX status, it may trigger ON anytime during the DRX cycle [6].

\section{UL retransmission timer}

According to 3GPP specifications, UE shall monitor PDCCH when it expects UL grant for retransmission. This functionality is implemented in the System Simulator using UL retransmission Timer. This timer is triggered 4 sub-frames after the uplink transmission.

\section{DL retransmission timer}

One retransmission timer is configured for every HARQ process in the downlink which means eight retransmission timers.Main purpose of this timer is to keep UE awake when it expects retransmissions on 
DL-SCH.Retransmission is triggered ON $8 \mathrm{~ms}$ after a downlink HARQ process is NACKED.The period of retransmission timer is kept long enough so that to avoid the case where during DL retransmission arrives but UE is sleeping [9].

\section{DRX inactivity timer}

It $s$ pecifies the number of consecutive PDCCH sub frames for which the UE should be Active after successfully decoding a PDCCH indicating a new transmission (UL or DL).

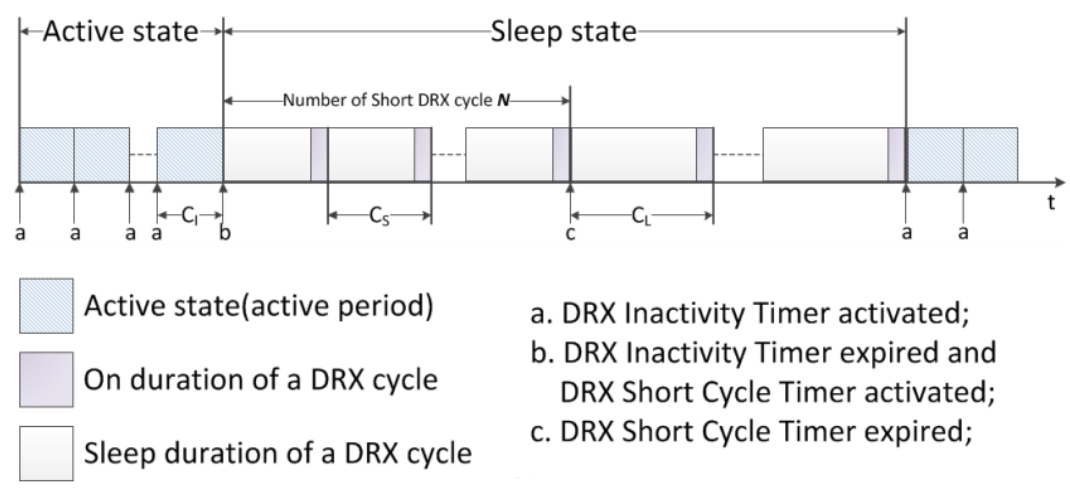

Figure 3: Different DRX cycles.

\section{Short DRX cycle}

It is the first type of DRX cycle (if configured) that needs to be followed when UE enters DRX mode. This IE indicates the length of the short cycle in sub frames which include ON time followed by a possible OFF (inactivity) time.

\section{Short DRX cycle timer}

It is expressed as multiples of short DRX-Cycle.The timer value can vary from 1 to 16 (short DRX cycles). This timer indicates the number of initial DRX cycles to follow the short DRX cycle before entering the long DRX cycle.

\section{Long DRX cycle}

A time span longer than the DRX Short Cycle where an UE stays in sleep state and wakes up only at the end of the cycle to monitor the PDCCH.

\section{On duration timer}

It specifies the number of consecutive PDCCH sub frames at the beginning of each DRX Cycle (DRX ON). i.e., is the number of sub frames over which the UE shall read PDCCH during every DRX cycle before entering the power saving mode (DRX OFF).

\section{DRX active timer}

Active Time is the time during which the UE is considered to be monitoring PDCCH. The Active Time includes the time while:

On Duration Timer is running, DRX Inactivity Timer is running and DRX Retransmission Timer is running.

Our proposed algorithm will follow markov chain process that will help to predict the dynamic value for DRX parameters. We give some overview of Markov Chain: 
Suppose there are two states. $\mathrm{A}=\mathrm{Off}$ states and $\mathrm{B}=\mathrm{On}$ states.If $\mathrm{A}$ has the average time of $10 \%$ and $\mathrm{B}$ has the average time of $90 \%$.

.2

.8 .4

.6

Figure 4: Markov Chain example.

If $\mathrm{A}$ has probability to come back to itself is $80 \%$ and probability to go to B state is $20 \%$. If $\mathrm{B}$ has the probability to come back to itself is $40 \%$ and go to state A is $60 \%$.

So the probability matrix will be $\mathrm{P}=$

$\left[\begin{array}{ll}.8 & a m p ; .2 \\ .6 & a m p ; .4\end{array}\right]$ and So $=[.1 .9]$

The next step of the Markov chain will be determined by

$\mathrm{S} 1=\mathrm{SoP}$

$=\left[\begin{array}{ll}.1 & .9\end{array}\right]$

$\left[\begin{array}{ll}.8 & a m p ; .2 \\ .6 & a m p ; .4\end{array}\right]$

$=[.62 .38]$

It is visible that the probability of being in state A is increasing and for B is decreasing.

$\mathrm{S} 2=\mathrm{S} 1 \mathrm{P}$

$=[.62 .38]$

$\left[\begin{array}{ll}.8 & a m p ; .2 \\ .6 & a m p ; .4\end{array}\right]$

$=[.724 .276]$

It is visible that the probability of being in state $\mathrm{A}$ is further increasing and for B is further decreasing.

$\mathrm{S} 3=\mathrm{S} 2 \mathrm{P}$

$=[.724 .276]$

$\left[\begin{array}{ll}.8 & a m p ; .2 \\ .6 & a m p ; .4\end{array}\right]$

$=[.7448 .2552]$

It is visible that the probability of being in state $\mathrm{A}$ is further increasing and for B is further decreasing. $\mathrm{S} 4=\mathrm{S} 3 \mathrm{P}$

$=[.7448 .2552]$

$\left[\begin{array}{ll}.8 & a m p ; .2 \\ .6 & a m p ; .4\end{array}\right]$

$=[.74896 .25104]$

It is visible that the probability of being in state A is further increasing and for B is further decreasing. $\mathrm{S} 5=\mathrm{S} 4 \mathrm{P}$ 
$=[.74892 .25104]$

$\left[\begin{array}{ll}.8 & a m p ; .2 \\ .6 & a m p ; .4\end{array}\right]$

$=[.74892 .25108]$

It is visible that the probability of being in state A is not so increasing and for B is not so decreasing.

$\mathrm{S} 6=\mathrm{S} 5 \mathrm{P}$

$=[.74892 .25108]$

$\left[\begin{array}{ll}.8 & a m p ; .2 \\ .6 & a m p ; .4\end{array}\right]$

$=[.75 .25]$

It is visible that the probability of being in state A is not increasing and for B is not further decreasing.

$\mathrm{S} 7=\mathrm{S} 6 \mathrm{P}$

$=[.75 .25]$

$\left[\begin{array}{ll}.8 & a m p ; .2 \\ .6 & a m p ; .4\end{array}\right]$

$=[.75 .25]$

It is visible that the probability of being in state $\mathrm{A}$ is not increasing at all and for B is not decreasing at all.

This is called stationary matrix because it is stationed at $\mathrm{P}=[.75 .25]$ and it is not changing as it is in steady mood. Thus, this process is called Markov process.

DRX allows an idle UE to power off the radio receiver for a predefined period(called the DRX cycle)instead of simultaneously listening to the radio channel.The system always transmits frames. The UE needs to be synchronized with every frame and wake up immediately before the transmission starts. When there are high traffic loads, there may not be any announce of arrival of traffic in UE so that no delay can be occurred.At a result the UE will consume extra unnecessary battery power.The DRX is applied then the UE is not forced to wake up at every announcement instead the UE disable the RF part to put the receiver in sleep for specific period of time and omit some of the announcement to reduce power consumption. When the UE wakes up then the UE needs to send a wake up frame to the network that its RF part has been enabled again and ready to receive frame that is distained for specific UE.To improve user equipment (UE)battery lifetime, LTE supports Discontinuous Reception(DRX)in both the RRC IDLE and the RRC CONNECTED radio resource control(RRC)states.DRX allows UEs which are not receiving data from their corresponding eNodeB (eNB)to monitor the physical downlink control channel (PDCCH)discontinuously. When UEs are not listening to the $\mathrm{PDCCH}$, they can enter a power saving mode in which most of their circuits can be turned off, thus reducing power consumption significantly.With DRX, the UE only wakes up periodically to listen to the PDCCH for a while, returning to the low power mode if no packet arrival is detected or resuming its normal operation in the case of new packet arrivals [10].

\section{Basic DRX operation}

When there is no data activity for drx-InactivityTimer amount of time (i.e., upon expiry of drxInactivityTimer) or DRX Command MAC CE is received,

- If the Short DRX cycle is configured, then the UE should start or restart drxShortCycleTimer and start using Short DRX Cycle.

- Else if Short DRX cycle is not configured, the UE should use the Long DRX cycle. 
- If drxShortCycleTimer expires, i.e., maximum number of short cycles are already used, then the UE should enter the Long DRX cycle.

- If drxShortCycleTimer expires, i.e., maximum number of short cycles are already used, then the UE should enter the Long DRX cycle.

- If a DRX Command MAC control element is received, the UE should stop onDurationTimer and drxInactivityTimer.

- If the Short DRX Cycle is used,

- $[(\mathrm{SFN} * 10)+$ subframe number $]$ modulo (shortDRX-Cycle) $=($ drxStartOffset) modulo (shortDRXCycle)

- else if the Long DRX Cycle is used

- $\left[\left(\mathrm{SFN}^{*} 10\right)+\right.$ subframe number $]$ modulo (longDRX-Cycle ) $=$ drxStartOffset

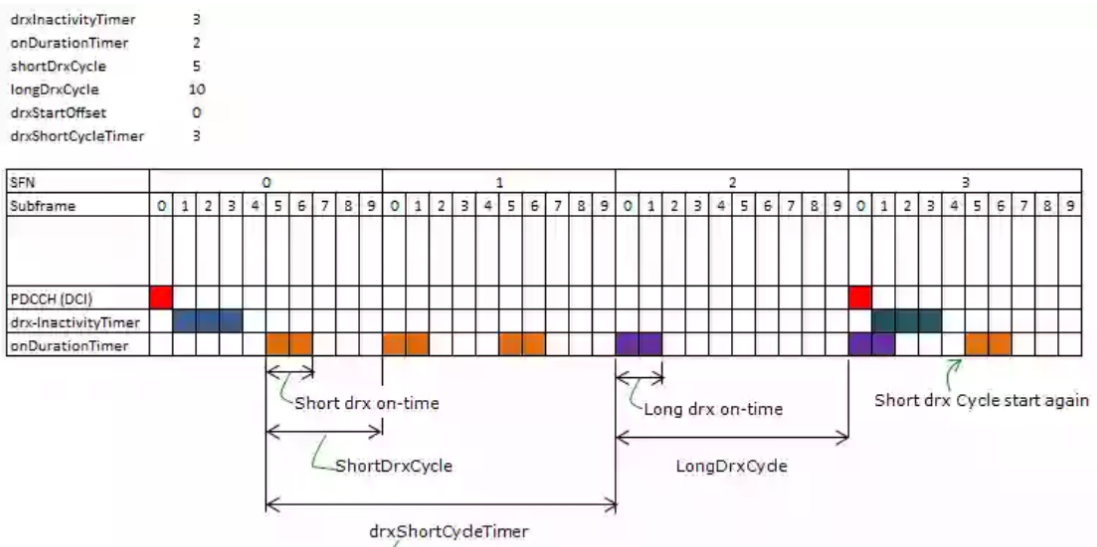

drxShortCydeTimer

(in subframe) $=$ shortDrxCycle $x$ drxShortcyde Timer

Figure 5: Basic DRX operation.

\section{Proposed algorithm:}

1: Repeat the procedure every $T$ ms.

3: Foreach user $i$ Do

4: If $M C S<=2$ Then

5: $T I N+=$ Step up IN

6: $T O N+=$ Step up $O N$

7: TS-=Step down ShortDrx

8: TL-=Step down LongDrx

9: Else If $M C S>=3$ and $M C S<=9$ Then

10: $T I N=I N$

11: $T O N=O N$

12: $T S=S$

13: $T L=L$

14: Else If $M C S>=10$ and $M C S<=19$ Then

15: $T I N-=$ Step down IN 
16: $T O N-=$ Step down $O N$

17: $T S+=$ Step up ShortDrx

18: $T L+=$ Step down LongDrx

19: Else If $M C S>=20$ and $M C S<=28$ Then

20: TIN $-=$ Step down IN

21: $T O N=O N$

22: $T S+=2 *$ Step up ShortDrx

23: $T L+=2 *$ Step down LongDrx

24: End for.

\section{Objective of the research}

To sum up, the current study is intended to answer the following major questions:

1.How much gain can be achieved in terms of battery life in UE using the proposed DRX model?

2. What is the impact of varying the proposed DRX model parameter settings and which settings show the best result? I.e.settings for On-duration Timer, Inactivity Timer, Tx value, Rx value etc.

3.What is the impact of the proposed DRX model on performance in terms of capacity, packet delay and packet loss?

4.How effective the proposed DRX model could be for the network by eliminating the unnecessary activity of UE?

\section{Methodology of the research}

We will develop a dynamic algorithm that satisfy the user requirement. We will discuss dynamic discontinuous reception(DRX)mechanism operation for UEs and eNodeBs.We will analyze which cqi value is best for idle time, Tx power, Rx power, sleep power, and burst length for UEs and eNodeBs. Then the results of these analysis will be calculated for battery consumption, lifetime length calculation and network load with specified scale.

We will take statistical data from different sources. We will search for best value of idle time, Tx power, $\mathrm{Rx}$ power, sleep power, and burst length from different trusted sources.

An adaptive DRX scheme that uses the monitored activity levels of the users to dynamically adjust the DRX parameters. The parameters are adjusted based on the data that is transmitted

between the UE and the eNB as well as the time at which data is transmitted.A threshold based adaptive system is presented in which the inactivity timer is adjusted based on the monitored CQI (Channel Quality Indicator).If the CQI is low, inactivity timer is increased; when the reported channel quality is good, inactivity timer is decreased.This leads to a balance between the power saving at the UE and the system throughput.An optimal algorithm to select the optimal DRX parameters depending on the delay constraints of the application and UE's power constraint is presented.Given a delay constraint, the algorithm allows the selection of optimal DRX parameters that maximize power saving and vice-versa.

\section{Flow chart for the research}

Figure 6: Flowchart for the research.

\section{Statistical tools to be used}

In this research we choose network simulation tool Network Simulator 3(NS-3), Matlab, Code Blocks, Sublime Text, Visual Trace Analyzer, NetSim and OMNeT ++ . 


\section{Possible outcome of the research}

We will develop a dynamic mechanism for DRX with similar and different input parameters to understand the optimality specified network with specified judging scale. The possible outcome of the research may show that the proposed dynamic mechanism with different cqi values for DRX may be better option for optimized battery consumption, efficient device lifetime and optimized network load. We may obtain optimized value for idle time, Tx power, Rx power, sleep power, and burst length for some particular cqi values.The algorithm will be presented in this research is different since they consider the observed application behavior to reduce the delay as well as reduce the energy consumption.Unlike the earlier techniques, these algorithms jointly consider the QoS parameters and the channel quality to improve the performance.

We will analyze simulation and analytical Results and how they change dynamically in accordance with cqi values given by PDCCH of UE's. Then we will analyze power consumption, which life time length is best for devices and how network will be optimized with the help of simulation with different parameters by using proposed dynamic DRX mechanism.

\section{Conclusion}

This research study a new dynamic discontinuous reception (DRX)mechanism operation for UEs and eNodeBs and provide better advantages to keep a balance between these different performance metrics that are battery consumption and increased latency. This research also investigates how parameters like idle time, Tx power, Rx power, sleep power, and burst length give dynamic solutions for the problem stated above. The approach will be validated against the simulation experiments. Several numerical examples will be presented to quantitatively show how the dynamic approach better than the other schemas.

Finally, our overall contribution is that we propose a better dynamic a schema for DRX.By this new proposed schema, we may have effective advantages, such as, may benefit the development of soon coming $5 \mathrm{G}$ networks and development of new and more optimized application in different quality networks.

\section{References}

[1] Scott Fowler, Mohammed Osman, Ahmed Omar Shahidullah "Analytical evaluation of extended DRX with additional active cycles for light traffic by Scott Fowler.", The International Journal of Computer and Telecommunications Networking archive, Volume 77, Issue: C ,2015.

[2] Ke Wang, Xi Li, Hong Ji, " Modeling and Optimizing the LTE Discontinuous Reception Mechanism Under Self-Similar Traffic.", IEEE Transactions on Vehicular Technology, Volume: 65, Issue: 7, July 2016.

[3] Shun-Ren Yang and Yi-Bing Lin, "Modeling UMTS Discontinuous Reception Mechanism by Shun-Ren Yang.", IEEE Transactions on Wireless Communications, Volume: 4, Issue: 1, Jan. 2005.

[4] Cheng-Wen Hsueh, "Analysis of Discontinuous Reception Power Saving with Radio Resource Control States Transition in LTE Networks.", on EuCNC,29 June-2 July 2015.

[5] Li-Ping Tung, Li-Chun Wang, Cheng-Wen Hsueh, Chung-Ju Chang, "Analysis of DRX power saving with RRC states transition in LTE networks", European Conference on Networks and Communications (EuCNC), Pages: $301-305,2015$.

[6] Songtao Gao and Hui Tian, Jianchi Zhu and Lan Chen "A More Power-Efficient Adaptive Discontinuous Reception Mechanism in LTE by Songtao Gao and Hui Tian."; Vehicular Technology Conference (VTC Fall), 2011 IEEE;01 December, 2011.

[7] Scott Fowler, Ranjeet S. Bhamber, Abdelhamid Mellouk . "Analysis of Adjustable and Fixed DRX Mechanism for Power Saving in LTE/LTE-Advanced Communications (ICC)", IEEE International Conference on 10-15 June, 2012.

[8] Chandra S. Bontu; Ed Illidge, "DRX mechanism for power saving in LTE" IEEE Communications Magazine, 26 June 2009. 
[9] Waqas Ahmad Khan , "Impact of DRX on VoIp performance and Battery Life in LTE" , LAP LAMBERT Academic Publishing,June 23, 2010.

[10] Sergio Herrer '1a-Alonso, Miguel Rodr'ıguez-P'erez, Manuel Fern'andez-Veiga and C'andido L'opezGarcia, "Adaptive DRX Scheme to Improve Energy Efficiency in LTE Networks with Bounded Delay", IEEE Journal on Selected Areas in Communications ,Volume: 33,pages: 2963 - 2973, Issue: 12 , Dec. 2015. 